Supporting Information: Nuclear Magnetic Resonance and Broadband Dielectric Spectroscopy Studies on the Dynamics of Ethylene Glycol in Mesoporous Silica

Melanie Reuhl, ${ }^{1}$ Max Weigler, ${ }^{1}$ Martin Brodrecht, ${ }^{2}$ Gerd Buntkowsky, ${ }^{2}$ and Michael Vogel $^{1}$

1) Institute of Condensed Matter Physics, Technische Universität Darmstadt, Hochschulstr. 6, 64289 Darmstadt, Germany

2) Eduard-Zintl-Institut für Anorganische und Physikalische Chemie,

Technische Universität Darmstadt, Alarich-Weiss-Str. 8, 64287 Darmstadt, Germany 


\section{MAGNETIZATION BUILDUP}

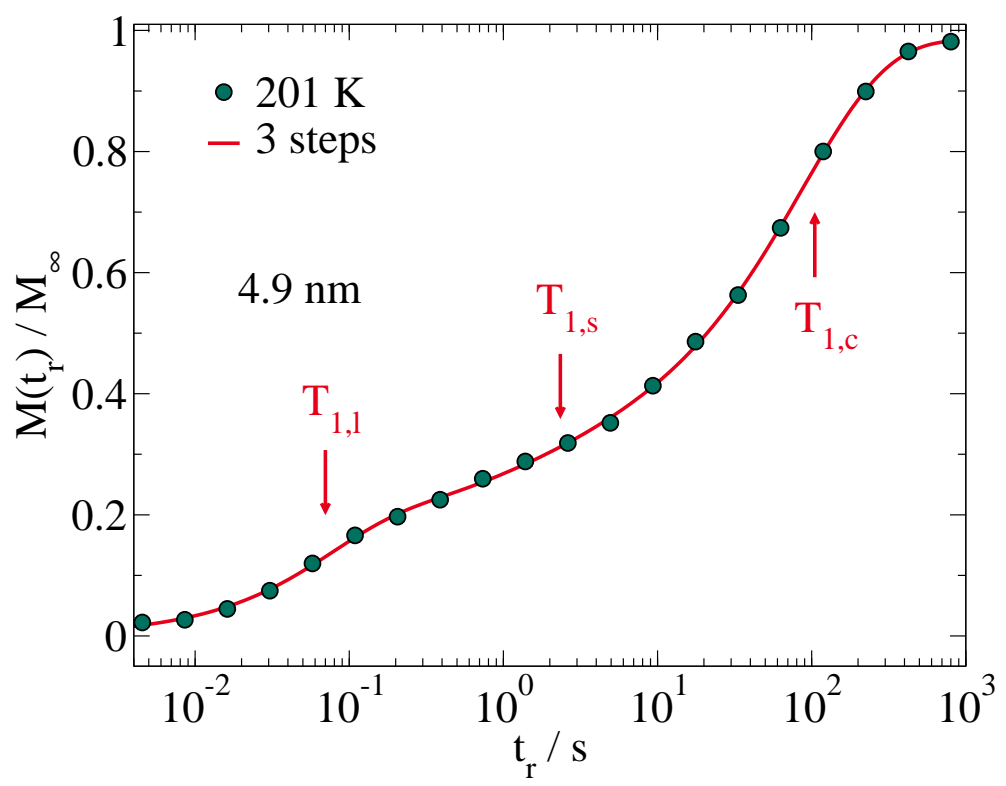

FIG. 1. Buildup of normalized ${ }^{2} \mathrm{H}$ magnetization $M\left(t_{\mathrm{r}}\right) / M_{\infty}$ for EG- $\mathrm{d}_{4}$ in silica pores with a diameter of $d=4.9 \mathrm{~nm}$ and $163 \%$ pore filling at $201 \mathrm{~K}$. The line is a fit to Eq. (7) of the main article using three SLR steps. The resulting SLR times $T_{1,1}, T_{1, \mathrm{~s}}$, and $T_{1, \mathrm{c}}$ are indicated.

Figure 1 shows an example of a ${ }^{2} \mathrm{H}$ magnetization buildup for a sample with overfilled pores in the deeply cooled temperature range. We find that ${ }^{2} \mathrm{H}$ spin-lattice relaxation occurs in three steps, which are related to liquid EG $\left(T_{1,1}\right)$, solid EG inside the pores $\left(T_{1, \mathrm{~s}}\right)$, and crystalline EG outside the pores $\left(T_{1, \mathrm{c}}\right)$. We see that internal and external EG fractions are clearly distinguishable. However, the intermediate step is small, which increases the statistical uncertainties of three-step fits. Therefore, the entire quantitative analyses in the main article are performed for samples, which do not have freezable excess EG so that two-step fits are sufficient. 


\section{BROADBAND DIELECTRIC SPECTROSCOPY}

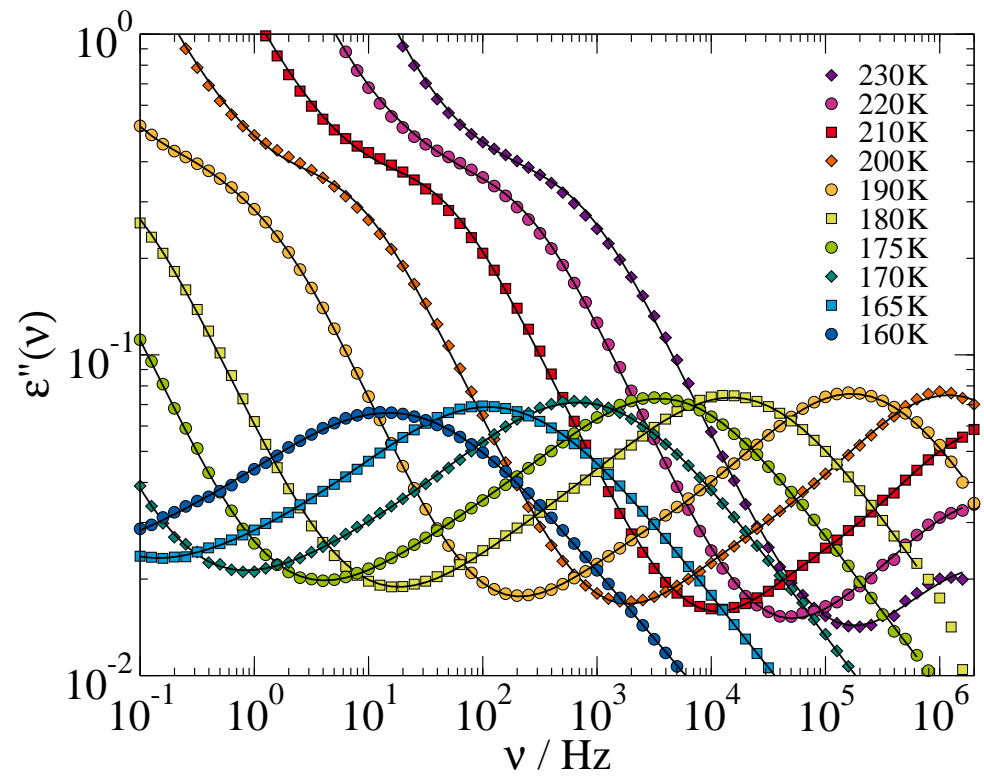

FIG. 2. Dielectric loss spectra $\varepsilon^{\prime \prime}(\nu)$ of EG-d $\mathrm{d}_{4}$ in silica pores with a diameter of $d=5.4 \mathrm{~nm}$ at various temperatures. The lines are fits to Eq. (3) of the main article.

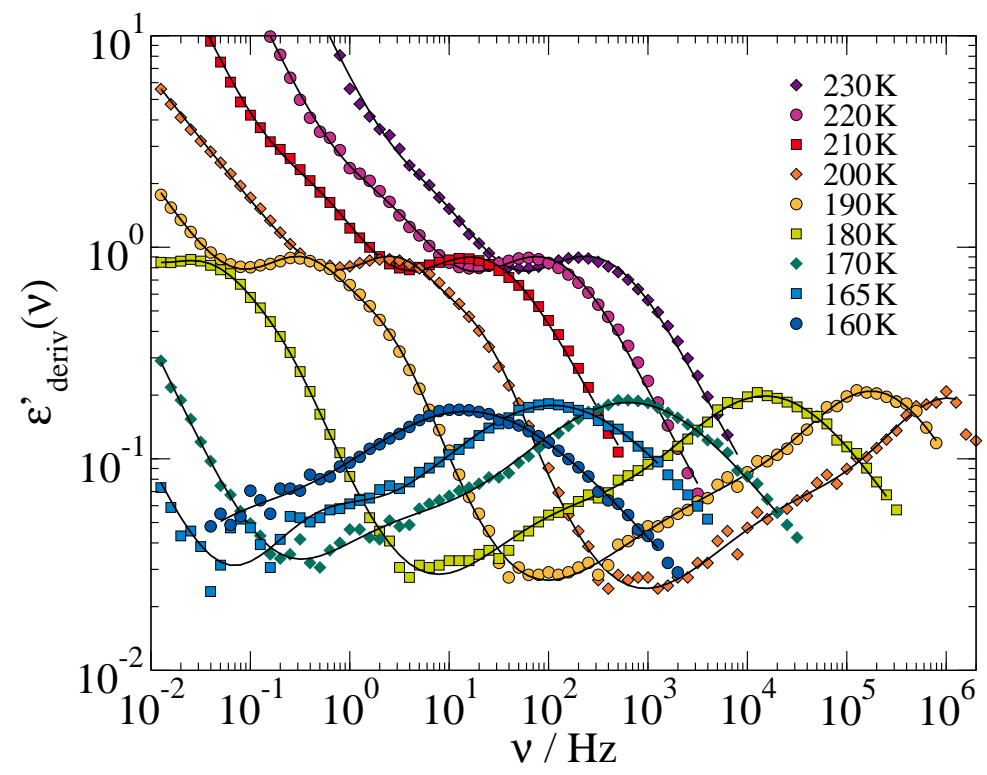

FIG. 3. Derivative of the real part $\varepsilon^{\prime}(\nu)$ of the dielectric permittivity of EG- $\mathrm{d}_{4}$ in silica pores with a diameter of $d=5.4 \mathrm{~nm}$ at various temperatures. The lines are fits to Eq. (3) of the main article. 
Results for the dielectric permittivity $\hat{\varepsilon}(\nu)$ of $\mathrm{EG}-\mathrm{d}_{4}$ in silica pores with a diameter of $d=5.4 \mathrm{~nm}$ at various temperatures are presented in Fig. 2 and Fig. 3. Specifically, the former graph displays the imaginary part $\varepsilon^{\prime \prime}(\nu)$, the dielectric loss, while the latter one depicts the derivative of the real part $\varepsilon^{\prime}(\nu)$. Fits to Eq. (3) of the main article reveal a conductivity contribution and three relaxation processes, which are denoted as P1, P2, and P4. Figure 4 illustrates the decomposition into these processes based on the dielectric loss $\varepsilon^{\prime \prime}(\nu)$ at $180 \mathrm{~K}$.

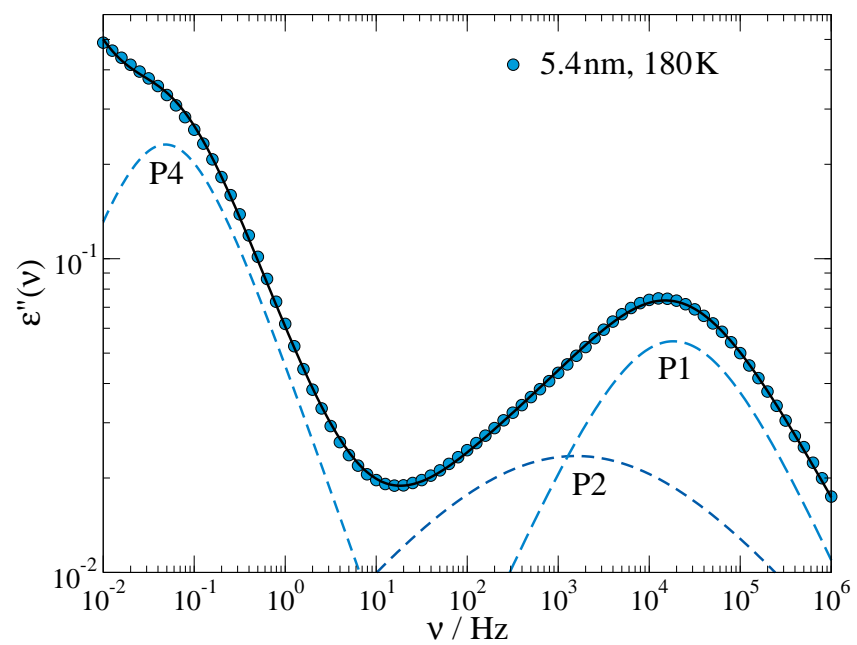

FIG. 4. Dielectric loss spectrum $\varepsilon^{\prime \prime}(\nu)$ of EG- $\mathrm{d}_{4}$ in silica pores with a diameter of $d=5.4 \mathrm{~nm}$ at $180 \mathrm{~K}$. The solid line is a fit to Eq. (3) of the main article. The dashed lines indicate the individual contributions of the relaxation processes $\mathrm{P} 1, \mathrm{P} 2$, and $\mathrm{P} 4$.

Figure 5 shows the temperature-dependent peak correlation times $\tau_{\mathrm{p}}$ resulting for the relaxation processes $\mathrm{P} 1, \mathrm{P} 2$, and $\mathrm{P} 4$ from these fits. We see that consistent correlation times are obtained when either interpolating $\varepsilon^{\prime}(\nu)$ and $\varepsilon^{\prime \prime}(\nu)$ simultaneously or fitting the derivative $\varepsilon_{\text {deriv }}^{\prime}(\nu)$. The microscopic origins of the relaxation processes $\mathrm{P} 1, \mathrm{P} 2$, and $\mathrm{P} 4$ are discussed in the main article. 


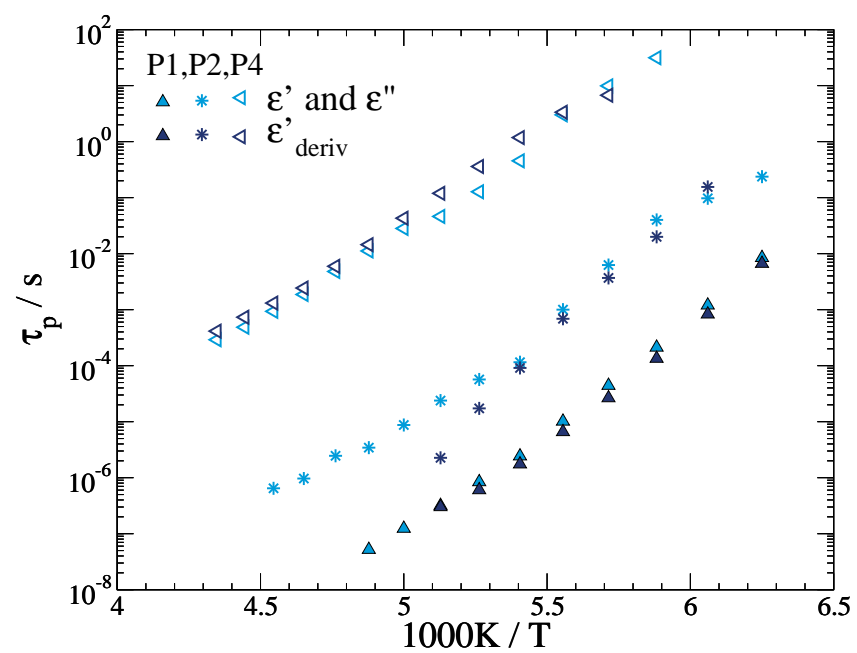

FIG. 5. Temperature-dependent peak correlation times $\tau_{\mathrm{p}}$ of the P1, P2, and P4 processes of EG- $\mathrm{d}_{4}$ in silica pores with a diameter of $d=5.4 \mathrm{~nm}$. Results obtained from fits of the complex dielectric permittivity $\hat{\varepsilon}(\omega)$, see Fig. 2 , and from fits of the derivative of the real part of the dielectric permittivity $\varepsilon^{\prime}(\nu)$, see Fig. 3, are compared.

Broadband dielectric spectroscopy (BDS) data for EG- $\mathrm{d}_{4}$ in silica pores with diameters of $d=2.4 \mathrm{~nm}$ and $d=9.2 \mathrm{~nm}$ can be found in the main article. Figure 6 displays the decomposition into the relaxation processes $\mathrm{P} 1, \mathrm{P} 2$, and $\mathrm{P} 4$ for the small pores and $190 \mathrm{~K}$.

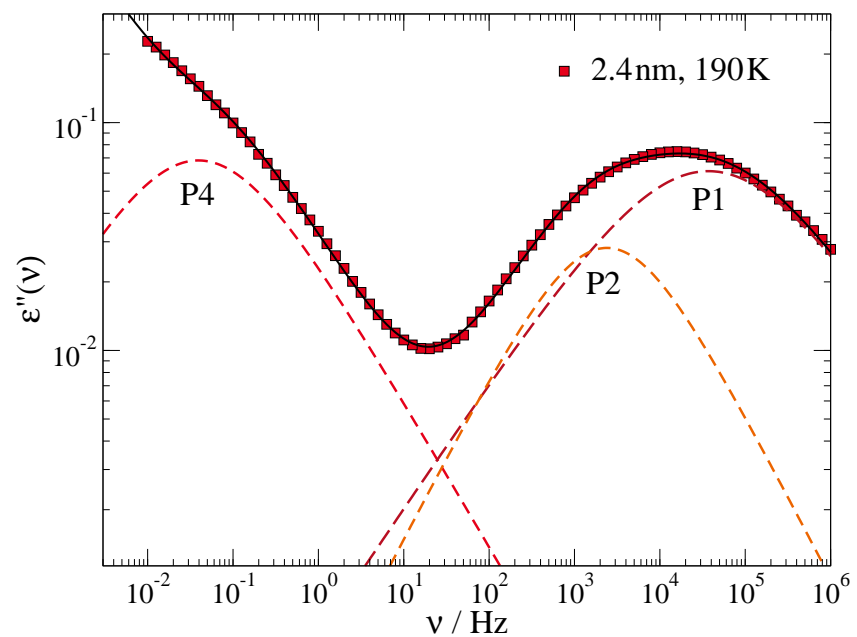

FIG. 6. Dielectric loss spectrum $\varepsilon^{\prime \prime}(\nu)$ of EG-d 4 in silica pores with a diameter of $d=2.4 \mathrm{~nm}$ at $190 \mathrm{~K}$. The lines are fits to Eq. (3) of the main article. The dashed lines indicate the individual contributions of the relaxation processes $\mathrm{P} 1, \mathrm{P} 2$, and $\mathrm{P} 4$.

In Figs. 7 and 8, we compare the relaxation times $\tau_{\mathrm{p}, n}$ and relaxation strengths $\Delta \varepsilon_{n}$ of 


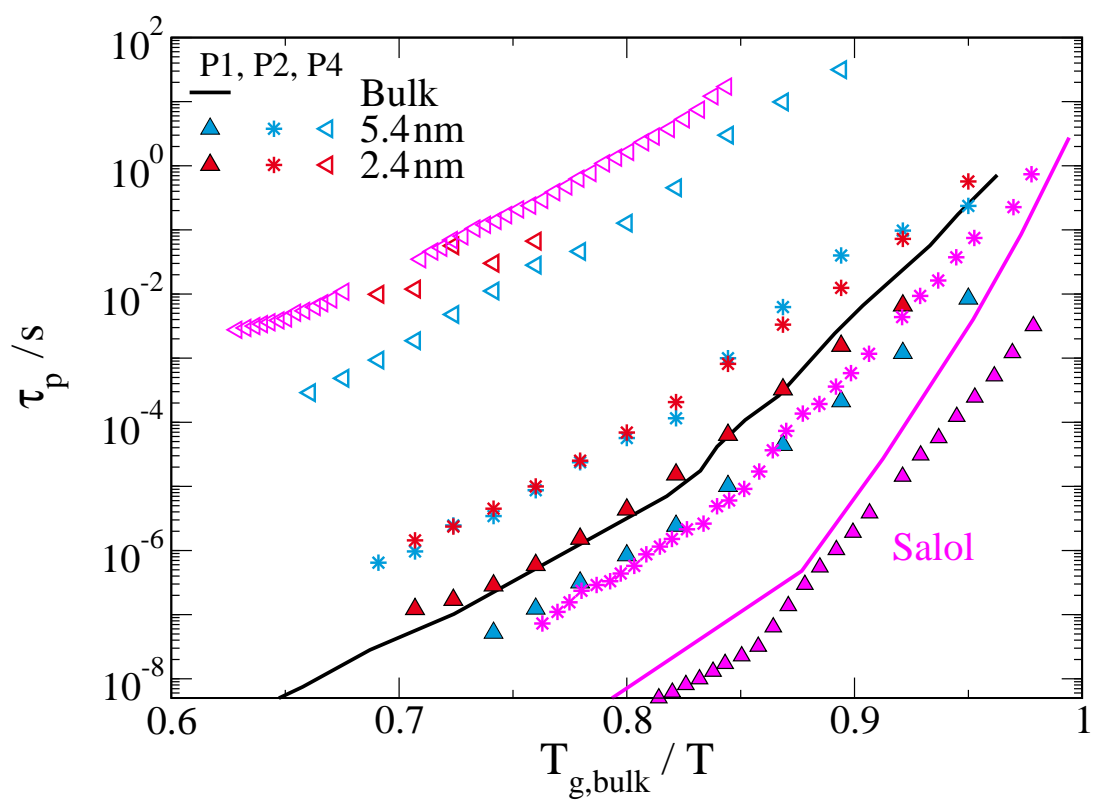

FIG. 7. Peak correlation times $\tau_{\mathrm{p}}$ of the BDS processes P1, P2, and P4 of EG- $\mathrm{d}_{4}$ in silica pores with diameters of $d=2.4 \mathrm{~nm}$ and $d=5.4 \mathrm{~nm}$ on a reduced temperature scale $T_{\mathrm{g}, \text { bulk }} / T$. Previous results $^{1,2}$ for the $\alpha$ process of bulk EG- $\mathrm{h}_{6}$ are shown as black solid line. For comparison, the corresponding data for salol in a controlled porous glass with a characteristic pore size of $7.5 \mathrm{~nm}$ are shown as magenta symbols. ${ }^{3}$ Moreover, the peak correlation times of the $\alpha$ process of bulk salol are included as magenta solid line. ${ }^{3}$ The bulk glass transition temperatures used for the scaling of the ordinate are $T_{\mathrm{g}, \mathrm{bulk}}=152 \mathrm{~K}$ for $\mathrm{EG}$ and $T_{\mathrm{g}, \mathrm{bulk}}=222 \mathrm{~K}$ for salol.

the BDS processes of EG in silica pores with the corresponding data for salol in a controlled porous glass. ${ }^{3}$ We observe several similarities: (i) P1 and P2, like the corresponding salol relaxations, have a similar but not identical temperature dependence. (ii) P1 agrees with the $\alpha$ process of the respective bulk liquid, but becomes faster near the glass transition temperature $T_{\mathrm{g}}$. (iii) P2 is slower at high temperatures, but approaches the bulk $\alpha$ process when cooling towards $T_{\mathrm{g}}$. (iv) P2 grows at the expense of P1 when decreasing the temperature in the deeply supercooled regime. For salol, this BDS phenomenology was rationalized using a core-shell model and assuming that an exchange of molecules between core and shell regions occurs on the time scale of the reorientation dynamics near $T_{\mathrm{g}}{ }^{3,4}$ Other common features of the BDS relaxation scenario of confined EG and confined salol are that (v) unlike the $\alpha$ processes of the bulk liquids, P1 and P2 broaden upon cooling, see Fig. 13(b) of the main article, and that P4, which was assigned to a Maxwell-Wagner process in the previous BDS 


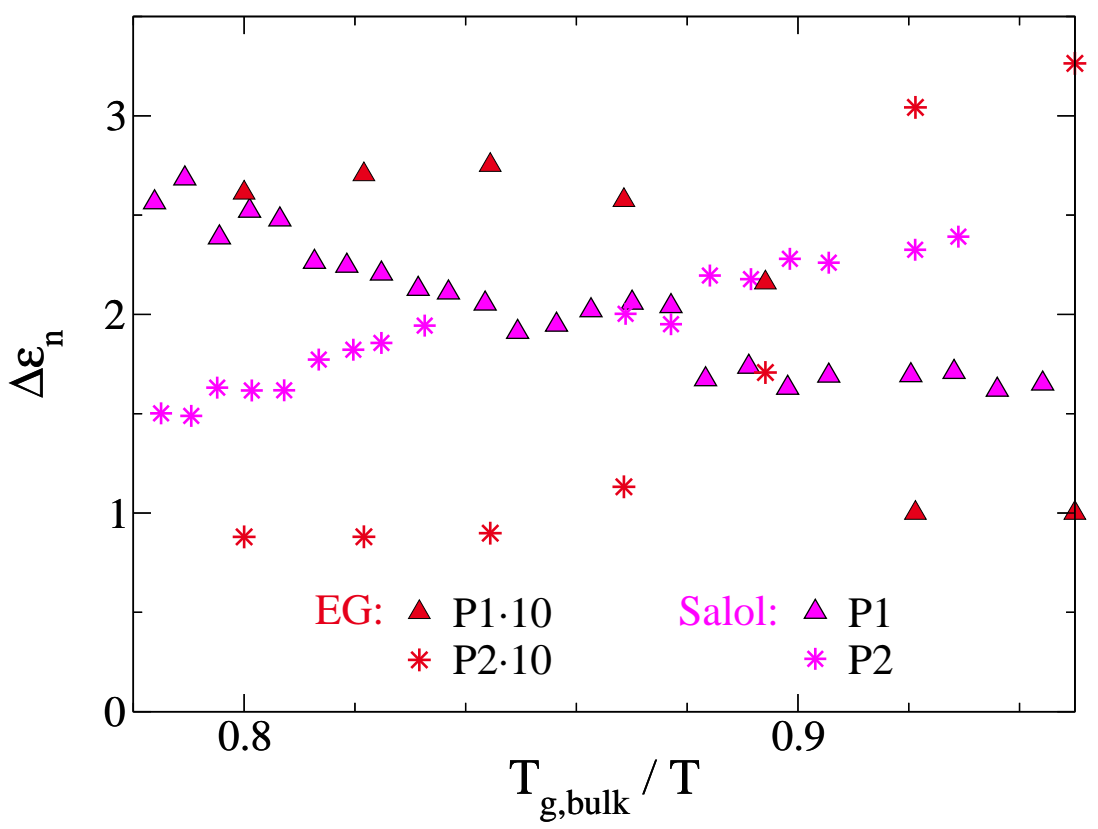

FIG. 8. Relaxation strengths $\Delta \varepsilon_{n}$ of the BDS processes $\mathrm{P} 1$ and P2 of EG- $\mathrm{d}_{4}$ in silica pores with a diameter of $d=2.4 \mathrm{~nm}$ on a reduced temperature scale $T_{\mathrm{g}, \text { bulk }} / T$. For comparison, the corresponding data for salol in a controlled porous glass with a characteristic pore size of $7.5 \mathrm{~nm}$ are shown as magenta symbols. ${ }^{3}$ For better comparability, the EG- $\mathrm{d}_{4}$ data are tenfold increased. The bulk glass transition temperatures used for the scaling of the ordinate are $T_{\mathrm{g}, \text { bulk }}=152 \mathrm{~K}$ for EG and $T_{\mathrm{g}, \mathrm{bulk}}=222 \mathrm{~K}$ for salol.

study, ${ }^{3}$ has similar time constants and temperatures dependence. Owing to these similarities, we assume that BDS findings of confined EG and confined salol can largely be explained on common grounds. 


\section{ANALYSIS OF SPIN-LATTICE RELAXATION TIMES}

In the high-temperature limit, i.e., when correlation times $\tau$ are much shorter than the inverse Larmor frequency $\omega_{\mathrm{L}},{ }^{2} \mathrm{H}$ SLR analysis can provide access to mean correlation times $\langle\tau\rangle$, largely independent of the shape of the spectral density $J_{2}(\omega)$. More precisely, in the limit $\omega_{\mathrm{L}} \tau \ll 1, T_{1}^{-1} \approx \frac{2}{3} \delta^{2} \tau$ applies to dynamics described by an exponential correlation function or, equivalently, a Lorentzian spectral density, where $\delta$ denotes the anisotropy parameter of the relevant quadrupolar coupling tensor. Thus, $T_{1}^{-1} \approx \frac{2}{3} \delta^{2}\langle\tau\rangle$ often holds when a distribution of correlation times $G(\log \tau)$ exists but dynamical exchange within this distribution still restores a common spin-lattice relaxation time $T_{1}$. However, a necessary condition for the validity of this relation between $T_{1}$ and $\langle\tau\rangle$ is that the long-time flank of $G(\log \tau)$ decays sufficiently fast such that all parts of the distribution obey $\omega_{\mathrm{L}} \tau \ll 1$. The latter condition is fulfilled for the distribution $G(\log \tau)$ associated with the Cole-Davidson spectral density, which has a long-time cutoff. ${ }^{5}$ Moreover, it is satisfied for logarithmic Gaussian distributions $G(\log \tau)$ with moderate width parameters $\sigma<3$, as obtained from

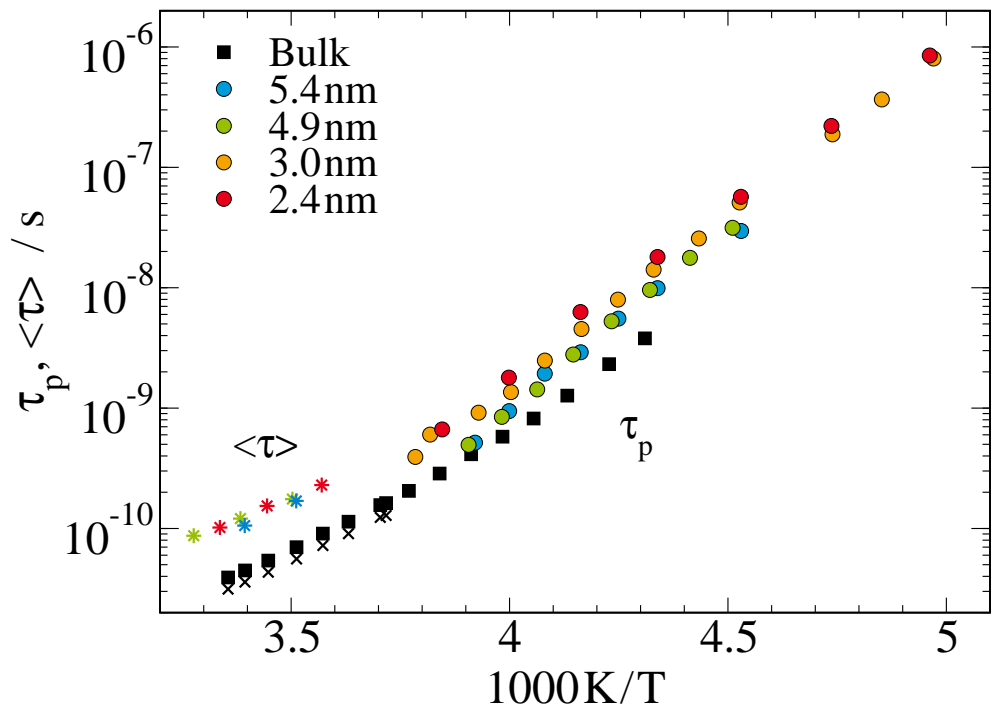

FIG. 9. Temperature-dependent correlation times obtained from ${ }^{2} \mathrm{H}$ SLR analysis for EG- $\mathrm{d}_{4}$ in the bulk liquid and EG- $\mathrm{d}_{4}$ in silica pores with the indicated diameters $d$ in the one-phase regime. The peak correlation times $\tau_{\mathrm{p}}$ are shown as squares (bulk) and circles (confinement), see Fig. 6 of the main article, while the mean correlation times $\langle\tau\rangle$ are displayed as crosses (bulk) and stars (confinement). 
numerical calculations. However, the condition is not met for the Cole-Cole spectral density used in the main article, which is linked to a distribution $G(\log \tau)$ with extended tails corresponding to power-law behaviors of the spectral density. ${ }^{5}$

Disregarding the latter issue, we use the relation $T_{1}^{-1} \approx \frac{2}{3} \delta^{2}\langle\tau\rangle$ together with the known anisotropy parameter $\delta=2 \pi \cdot 125 \mathrm{kHz}$ to translate the high-temperature $T_{1}$ values of confined and bulk EG- $\mathrm{d}_{4}$ reported in the main article into mean correlation times $\langle\tau\rangle$. In Fig. 9 , we compare the peak correlation times $\tau_{\mathrm{p}}$ determined in the main article with these mean correlation times $\langle\tau\rangle$. As the mean correlation times correspond to a time average, they are governed by the longest correlation times of a distribution and, hence, they can be longer than the peak correlation times discussed in the main article if dynamical heterogeneities exist. ${ }^{6}$ In Fig. 9, we see that this effect is not relevant for bulk EG, which is described by the CD distribution with a long-time cutoff. Specifically, there is only the minor difference between mean and peak correlation times expected for this distribution. Contrarily, owing to significant low-frequency and, thus, long-time contributions to the dielectric loss spectra of confined EG, we expect that the mean correlation times $\langle\tau\rangle$ are longer than the peak correlation times $\tau_{\mathrm{p}}$ for these samples. This expectation is supported by the observation that the difference between the $\langle\tau\rangle$ values of bulk and confined EG is larger than the discrepancy between the $\tau_{\mathrm{p}}$ values of these samples but we cannot arrive at an unambiguous statement because the temperature ranges accessible to the $\tau_{\mathrm{p}}$ and $\langle\tau\rangle$ analyses do not overlap for confined EG. Either way, the mean correlation times suggest that EG dynamics is independent of the pore diameter in the range $d=2.4-5.4 \mathrm{~nm}$, see Fig. 9, while the positions of the $T_{1}$ minima and the self diffusion coefficients $D$ in the main article clearly revealed a slowdown in narrower pores. Most probably, the analysis of the mean correlation times misses this important effect because the value of $\langle\tau\rangle$ is, at least for small and medium-sized pores, dominated by the largely pore-size independent correlation times of slow EG species near the pore walls. This is one of the reasons why peak correlation times $\tau_{\mathrm{p}}$ are considered throughout the main article.

Finally, we compare measured and calculated ${ }^{2} \mathrm{H}$ SLR times $\left\langle T_{1, n}\right\rangle$ of confined EG- $\mathrm{d}_{4}$ in Fig. 10. Specifically, the experimental results for $\left\langle T_{1, f}\right\rangle$ and $\left\langle T_{1, s}\right\rangle$ determined from the fast and slow SLR steps in the main article, respectively, are compared with data calculated from the BDS processes P1 and P2. Relating to $\left\langle T_{1, f}\right\rangle$, we find agreement between the measurements and the expectations for P1 for the small and medium-sized pores and moderate 


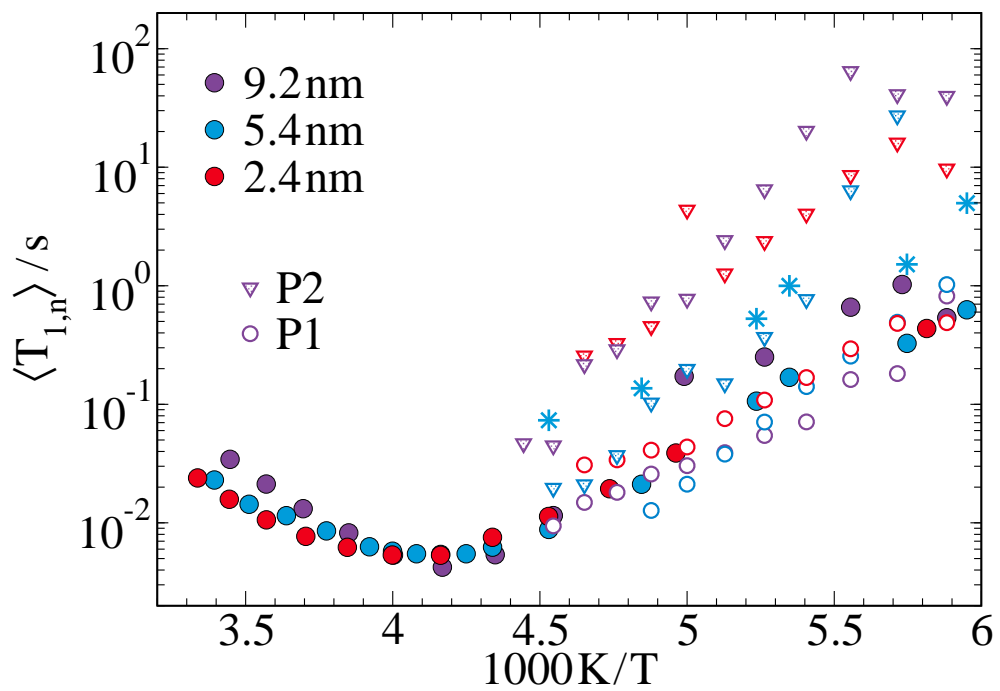

FIG. 10. Temperature-dependent mean ${ }^{2} \mathrm{H}$ SLR times $\left\langle T_{1, n}\right\rangle$ of EG-d4 in pores with the indicated diameters $d$ at $\sim 100 \%$ filling. The experimental results $\left\langle T_{1, f}\right\rangle$ (solid circles) and $\left\langle T_{1, s}\right\rangle$ (stars) determined from the fast and slow SLR steps, respectively, are compared with data calculated from the BDS processes P1 (open circles) and P2 (shaded triangles). For the calculations, we transform the CC susceptibilities obtained for P1 and P2 from the fits of the measured complex dielectric permittivities $\hat{\varepsilon}(\omega)$ into the corresponding CC spectral densities $J_{2}(\omega)$ and use the latter together with $\delta=2 \pi \cdot 125 \mathrm{kHz}$ and $\omega_{\mathrm{L}}=2 \pi \cdot 46.1 \mathrm{MHz}$ in Eq. (9) of the main article. Strictly speaking, this approach assumes exponential SLR. Hence, it is valid for the fast step, while it neglects the nonexponentiality of the slow one.

deviations for the large pores, supporting the conclusion that the fast SLR step and P1 probe the dynamics of the liquid EG fraction in the silica pores. Relating to $\left\langle T_{1, s}\right\rangle$, we find, however, no satisfactory agreement between the measured and any of the calculated data.

\section{REFERENCES}

${ }^{1}$ A. Huwe, F. Kremer, and P. Behrens, Phys. Rev. Lett. 82, 2338 (1999).

${ }^{2}$ A. Huwe, F. Kremer, J. Kärger, P. Behrens, W. Schieger, G. Ihlein, Ö. Weiß, and F. Schüth, J. Molec. Liqu. 86, 173 (2000).

${ }^{3}$ M. Arndt, R. Stannarius, W. Gorbatschow, and F. Kremer, Phys. Rev. E 54, 5377 (1996).

${ }^{4}$ R. Stannarius, F. Kremer, and M. Arndt, Phys. Rev. Lett. 75, 4698 (1995).

${ }^{5}$ P. A. Beckmann, Phys. Rep. 171, 85 (1988). 
${ }^{6}$ M. Vogel, C. Brinkmann, H. Eckert, and A. Heuer, Phys. Chem. Chem. Phys. 4, 3237 (2002). 